\title{
Exposure to bisphosphonates and risk of common non-gastrointestinal cancers: series of nested case-control studies using two primary-care databases
}

\author{
Y Vinogradova*, ${ }^{*}$, C Coupland ${ }^{1}$ and J Hippisley-Cox ${ }^{1}$ \\ ${ }^{1}$ Division of Primary Care, 13th floor, Tower Building, University Park, Nottingham NG2 7RD, UK
}

Background: Bisphosphonates are the most commonly prescribed osteoporosis drugs but long-term effects are unclear, although antitumour properties are known from preclinical studies.

Methods: Nested case-control studies were conducted to investigate bisphosphonate use and risks of common nongastrointestinal cancers (breast, prostate, lung, bladder, melanoma, ovarian, pancreas, uterus and cervical). Patients 50 years and older, diagnosed with primary cancers between 1997 and 2011, were matched to five controls using the UK practice-based QResearch and Clinical Practice Research Datalink (CPRD) databases. The databases were analysed separately and the results combined.

Results: A total of 91556 and 88845 cases were identified from QResearch and CPRD, respectively. Bisphosphonate use was associated with reduced risks of breast (odds ratio (OR): 0.92, 95\% confidence interval (Cl): 0.87-0.97), prostate (OR: 0.87, 95\% Cl: 0.79-0.96) and pancreatic (OR: 0.79,95\% Cl: 0.68-0.93) cancers in the combined analyses, but no significant trends with duration. For alendronate, reduced risk associations were found for prostate cancer in the QResearch (OR: 0.81, 95\% Cl: 0.70-0.93) and combined (OR: 0.84, 95\% Cl: 0.75-0.93) analyses (trend with duration P-values 0.009 and 0.001). There were no significant associations from any of the other analyses.

Conclusion: In this series of large population-based case-control studies, bisphosphonate use was not associated with increased risks for any common non-gastrointestinal cancers.

Bisphosphonates were introduced as a treatment for postmenopausal osteoporosis (National Institute for Health and Clinical Excellence, 2008a, b) in the 1990s, and prescribing has substantially increased (Kanis et al, 2008). The effects of bisphosphonates are long term as the drugs accumulate in bones and are released for several years after treatment ends (Watts and Diab, 2010). It is a relatively new treatment and very few studies have looked at the long-term effect of bisphosphonates on risks of different cancers in the general population.

Although antitumour properties of bisphosphonates have been discovered in preclinical studies (Croucher et al, 2003; Guise, 2008) and reaffirmed in the treatment of bone metastases (Gnant, 2010), no long-term randomised clinical trials have been run to determine the effect of bisphosphonates on cancer incidence. Epidemiological studies have consistently reported a reduced risk of breast cancer in bisphosphonate users (Chlebowski et al, 2010; Newcomb et al, 2010; Rennert et al, 2010; Vestergaard et al, 2011; Cardwell et al, 2012), but the effects of bisphosphonates on other common nongastrointestinal cancers are still uncertain, having been investigated in only one up-to-date observational study using the Clinical Practice Research Datalink (CPRD) (Cardwell et al, 2012). Previous studies have generally been insufficiently powered to

*Correspondence: Y Vinogradova; E-mail: yana.vinogradova@nottingham.ac.uk

Received 16 April 2013; revised 5 June 2013; accepted 11 June 2013; published online 18 July 2013 
detect associations for other types of cancer, and few have looked at associations with individual types of bisphosphonate drugs, and so there is little data to establish definitive conclusions. Our aim, therefore, was to investigate the associations between bisphosphonates and risks of common cancers in the general population using a nested case-control design and including all available data from two large primary-care databases - both the QResearch and the CPRD.

\section{MATERIALS AND METHODS}

Data source. The two largest primary-care databases in the UK, QResearch and CPRD, were used. Each database covers around 6\% of the UK population from more than 600 general practices, and contains electronic records including patient demographics, referrals, tests and prescriptions. Both are representative of the general population in the United Kingdom, have been rigorously validated using other sources of information (Jick et al, 2003; Hippisley-Cox et al, 2004) and have been used for a range of safety studies involving commonly prescribed medications (Green et al, 2010; Hippisley-Cox and Coupland, 2010; Parker et al, 2010; Walker et al, 2011). The protocol for this study was published in Vinogradova et al (2012) and, although originally based on QResearch, a replicate study has also been conducted using CPRD to examine any possible differences between the two and further increase in the statistical power by running analyses to derive combined results from both data sources.

Study design. Open cohorts of patients were identified in each database: patients were aged 50 years and older and registered with the practice at some time during the study period between January 1997 and July 2011. For this paper, we selected the most common solid, non-gastrointestinal cancers (breast, prostate, lung, bladder, pancreatic, ovarian and melanoma) as the outcomes and identified incident cases from the cohorts. Less common female cancers (cervix and uterus) were also considered. Each case was matched to up to five controls by age, sex, practice and calendar year. All controls were alive and registered with the practice at the date of the first recorded diagnosis of cancer in their matched case, which we defined as the index date for each case and their matched controls. For cases and controls, patients were included only if they had at least 2 years of data before their index date. Cases and controls with bisphosphonate prescriptions licensed for any malignancies before the index date (date of diagnosis for cases or equivalent date for controls) were excluded. For breast cancer, male patients and patients with a record of mastectomy before their first prescription of bisphosphonates were excluded. Patients with Paget's disease were also excluded.

Exposure to bisphosphonates. Exposure to bisphosphonates was assessed, including prescriptions for alendronate, etidronate, ibandronate and risedronate as the nationally licensed drugs for the treatment of osteoporosis (BNF 6.6.2) (British Medical Association and Royal Pharmaceutical Society of Great Britain, 2008). Information was extracted on all prescriptions for bisphosphonates during the observation period - defined as a period between the date of patient registration with the general practice and 6 months before the index date. Prescriptions in the past 6 months before the index date were not used to reduce protopathic bias because early symptoms of cancer such as low weight or bone ache could lead to bisphosphonate treatments. For the main analyses, a patient was considered to be a bisphosphonate user if they had at least one prescription during the observation period. Cumulative exposure was estimated by summing the durations of all bisphosphonate prescriptions for each patient, considering gaps of fewer than 90 days between two prescriptions as continuous therapy. Duration of exposure to bisphosphonates was analysed using the following categorisations: no use; short-term use (used for $<1$ year); long-term use (used for $>1$ year). For analyses of the most common cancers (breast, prostate, lung, bladder and melanoma), finer categories for duration of use of bisphosphonates were considered: no use, $<6$ months; $7-36$ months; 37-72 months; and 73 months or more. A test for trend was performed using the actual number of months.

Confounding variables. All the analyses included potential confounders established as risk factors for cancer. Body mass index (BMI) (Henderson and Bernstein, 2008), a continuous variable, was based on values recorded at the date closest to 1 year before the index date. Using Read codes, smoking status (Hecht, 2008) (current smoker - light (1-9 cigarettes per day), medium (10-19), heavy (20 or more), ex-smoker, non-smoker); alcohol consumption (Schütze et al, 2011); and ethnicity (Ferlay et al, 2010) (White or not recorded, Black, Asian, Other) used values recorded at the closest date before the index date. The analysis also adjusted for history of osteoporosis (McGlynn et al, 2008), including diagnosis of osteoporosis or osteopenia or previous fractures (recorded before the index date); use of drugs increasing risks of fracture and cancer such as systemic corticosteroids and acid-suppressive medications (including $\mathrm{H} 2$ antagonists (BNF 1.3.1), proton pump inhibitors (BNF 1.3.5) and antacids (BNF 1.1.1)); (Corley et al, 2010) use of anti-inflammatory drugs (Coussens and Werb, 2002) (traditional non-steroidal antiinflammatory drugs, cyclo-oxygenase 2 inhibitors and aspirin); (Gonzalez-Perez et al, 2003) and use of vitamin D (Mocellin, 2011) if they were prescribed at least 1 year before the index date. For female cancer patients, use of hormone replacement therapy and oral contraceptive pills were also added to the analyses.

If they were diagnosed at least a year before the index date, comorbidities, which affect risks of cancer, were also included: rheumatoid arthritis (Thomas et al, 2000) for any cancer; benign breast disease for breast cancer; diabetes for pancreatic (Vincent et al, 2011) and uterine (Burbos et al, 2010) cancers; hypertension for uterine cancer (Bangalore et al, 2011); and gastrointestinal disorders for pancreatic cancer (Vinogradova et al, 2012). The results were also adjusted for cancer-specific family histories of cancer (Mai et al, 2011) (to reduce recall bias in cases only if recorded at least 6 months before the index date; Chang et al, 2006).

Statistical analysis. This study used conditional logistic regression to estimate odds ratios with $95 \%$ CIs for cancers at each selected site. The Wald test was used for estimating the effects of duration and testing for differences between bisphosphonate types. Missing values for the confounding factors (BMI, smoking status and alcohol intake) were imputed using ICE multiple imputation program in Stata (StataCorp LP, College Station, TX, USA) (Royston, 2005) where all the confounding variables and exposure to bisphosphonates were included into the models. Ten imputed data sets were created and the results were combined using Rubin's rules (Royston, 2004).

Each database was analysed separately and the results combined using the method of Mantel and Haenszel for fixed-effect models.

The primary analyses were based on bisphosphonate exposure excluding prescriptions in the 6 months before the index date. Five sensitivity analyses were carried out for each data set. The first was to eliminate possible bias by redefining bisphosphonate exposure as at least two prescriptions and considering patients with one prescription only as non-users. Such patients might never have started treatment or have soon stopped it because of adverse gastrointestinal effects. The second sensitivity analysis was based on all prescriptions before the index date including the ones issued in the past 6 months. This aimed to eliminate another form of bias caused by a possible oversampling of unexposed cases and controls in the main analyses. The third sensitivity analysis was run on 
patients with at least 6 years of medical records and redefined exposure based on prescription information only between 72 and 6 months before the index date. This was to eliminate possible bias arising from the different observation times for patients in the main analyses.

Townsend scores, a measure of deprivation, were available for only $49 \%$ of CPRD practices, and only $36 \%$ of CPRD patients had it recorded. Deprivation, therefore, was not included as a confounding variable in any of the main analyses. The fourth sensitivity analysis included Townsend scores as a confounding variable, but was run only on patients with a valid code. The fifth and final sensitivity analysis was run on observations with recorded values for BMI, smoking status and alcohol consumption. For the fourth and fifth sensitivity analyses, the definitions for use of bisphosphonates and years of medical records were identical to the main analysis.

Although sample size calculations were carried out and presented in the protocol (Vinogradova et al, 2012), all available data were used in the analyses. We considered a $1 \%$ level as statistically significant to allow for multiple comparisons, but have presented $95 \%$ CIs in our results to create parity with other studies. Stata Version 12 was used for all analyses.

\section{RESULTS}

Study population. Within the study period, we identified 91556 and 88845 cases of cancers of interest in people aged 50 years and older from QResearch and CPRD, respectively. These were matched to 427674 and 415583 controls, respectively, all with at least 2 years of medical records (Figure 1). Median years of available records was 17 (interquartile range 10-28 in QResearch cases and controls, 10-29 in CPRD cases and 10-30 CPRD controls), and it was consistent both for cancer sites and for cases and controls. Table 1 shows the numbers of cases and controls for each cancer site and also the characteristics of the study population. QResearch and CPRD provided similar samples from the general population. The QResearch cases and controls were about the same age (mean 69.4 years, standard deviation 9.8 in cases; 69.4 years, 9.7 in controls) as CPRD (69.9 years, 10.4 in cases; 69.7 years, 10.2 in controls) but included fewer women ( $48 \%$ vs $51 \%$ ), and so had slightly different incidences in cancers of interest.

The proportion of bisphosphonate users (4.2\%) was the same in both databases and in cases and controls, with similar proportions of patients prescribed different types of bisphosphonates. Figure 2 shows that in both databases the proportion of cases and controls with at least one bisphosphonate prescription consistently grew from $1 \%$ in 1997 to $7 \%$ in 2011. Use of etidronate decreased but use of alendronate and risedronate increased, for alendronate reaching over $80 \%$ for bisphosphonate users with at least one prescription. Most bisphosphonate users were older than 60 years (92\% in both databases) and their median duration of use was 20 months (interquartile range 7-43) in cases and controls in QResearch and 19 months (6-40 in cases and 6-41 in controls) in CPRD. The median duration varied slightly between the cancer sites, from 16.5 months for cervix cases and 27 for melanoma cases in QResearch, and 17 for lung cancer cases to 22.5 for melanoma cases in CPRD. A much higher proportion of women were users than men $(6.6 \%$ women $v s 2.0 \%$ men in cases, $6.8 \%$ women $v s 1.7 \%$ men in controls for QResearch, 6.5\% women $v s 1.9 \%$ men in cases and $6.6 \%$ women vs $1.7 \%$ men in controls for CPRD). Use of bisphosphonates did not necessarily follow a recorded diagnosis of osteoporosis/osteopenia or a history of fractures; over $30 \%$ of bisphosphonate users did not have either factor recorded
(38\% cases and 36\% controls in QResearch and 36\% cases and $34 \%$ controls for CPRD).

Table 2 shows the number and proportion of cases and controls who were bisphosphonate users for each cancer site and overall use of the drug by database. Figure 3 contains the results of combined analyses for overall bisphosphonate use. Table 3 presents trend test results along with odds ratios for short- and long-term use for the seven most common cancers. Table 4 shows further analyses for the associations of different types of bisphosphonate use with breast, prostate and lung cancer risks.

Breast cancer. Overall use of bisphosphonates was associated with a reduced risk of breast cancer but it was only significant in QResearch analyses (adjusted odds ratio (AOR): 0.89, 95\% CI: 0.82-0.97) and the combined analyses (AOR: 0.92, 95\% CI: 0.87-0.98). Clinical Practice Research Datalink analyses demonstrated a similar direction of the association (AOR: 0.95, 95\% CI: $0.88-1.03)$, but it was not statistically significant $(P=0.2)$. None of the associations were duration-dependent, with the only statistically significantly decreased risk for QResearch $(P=0.008)$ in the subcategory between 7 and 36 months (AOR: $0.87,95 \% \mathrm{CI}$ : 0.78-0.96 for QResearch; $0.95,0.86-1.05$ for CPRD; 0.91, 0.85-0.98 for combined). The risks did not vary between bisphosphonate types and none of them had statistically significant associations with breast cancer risk.

The sensitivity analysis, which defined bisphosphonate use as at least two prescriptions, demonstrated an even stronger reduced association with breast cancer risk (AOR: 0.87, 95\% CI: 0.80-0.94, $P<0.001$ for QResearch; 0.94, 0.87-1.01, $P=0.1$ for CPRD; 0.90, $0.85-0.96, P<0.001$ for combined), but it remained statistically significant only for short-term use, and only for QResearch and combined (AOR: 0.80, 95\% CI: 0.71-0.91, $P<0.001$ for QResearch; $0.87,0.80-0.94, P<0.001$ for combined) but not for CPRD (AOR: $0.92,95 \%$ CI: 0.82-.03). There was no significant association with long-term use for any analyses.

Other female cancers. Although QResearch analyses for ovarian cancer showed an almost $20 \%$ increased, but not statistically significant, risk (AOR: 1.19, 95\% CI: 0.96-1.47), CPRD analyses had an opposite, and also not statistically significant, association (AOR: 0.84, 95\% CI: 0.67-1.04), with combined results showing no association at all (AOR: 1.00, 95\% CI: 0.86-1.16). None of the results from the different databases were duration-dependent.

There was a significant association between risedronate use and ovarian cancer risk for QResearch (24 exposed cases) and the combined analyses (56 exposed cases) (AOR: 0.48, 95\% CI: $0.31-0.75, P=0.001$ for QResearch; $0.62,0.46-0.84, P=0.002$ for combined). The CPRD analysis (32 exposed cases) showed a reduced risk but not statistically significantly (AOR: 0.77, 95\% CI: $0.52-1.16)$. The trend tests were not statistically significant ( $P=0.013$ for QResearch; $P=0.6$ for CPRD; $P=0.07$ for combined) and this was consistent across all sensitivity analyses.

Overall use of bisphosphonates was not associated with cervical or uterine cancer risk for either database or for the combined analyses. Further analyses demonstrated no associations with duration of use.

Prostate cancer. Adjusted analyses in both databases demonstrated a reduced risk associated with bisphosphonate use but not significant (AOR: 0.90, 95\% CI: 0.79-1.02 for QResearch; 0.84, 0.73-0.96 for CPRD), but contributing to a significant association in the combined analysis (AOR: 0.87; 95\% CI: 0.79-0.96). Although neither of the databases showed a significant durationdependent association (QResearch $P_{\text {trend }}=0.064 ; \quad \mathrm{CPRD}$ $\left.P_{\text {trend }}=0.03\right)$, the combined analyses did $\left(P_{\text {trend }}=0.005\right)$, with a $15 \%$ reduced risk associated with long-term use of bisphosphonates (AOR: 0.85,; 95\% CI: 0.76-0.95). 


\section{QRESEARCH}

Cancers of interest 1997-2011, cases and controls with at least 2 years of data

Total: 92159 and 431730

Breast: 24891 and 116355

Prostate: 26656 and 125046

Lung: 18019 and 84428

Bladder: 7485 and 34971

Melanoma: 5008 and 23489

Ovary: 3431 and 16127

Pancreas: 3597 and 16870

Uterus: 2250 and 10595

Cervix: 822 and 3849

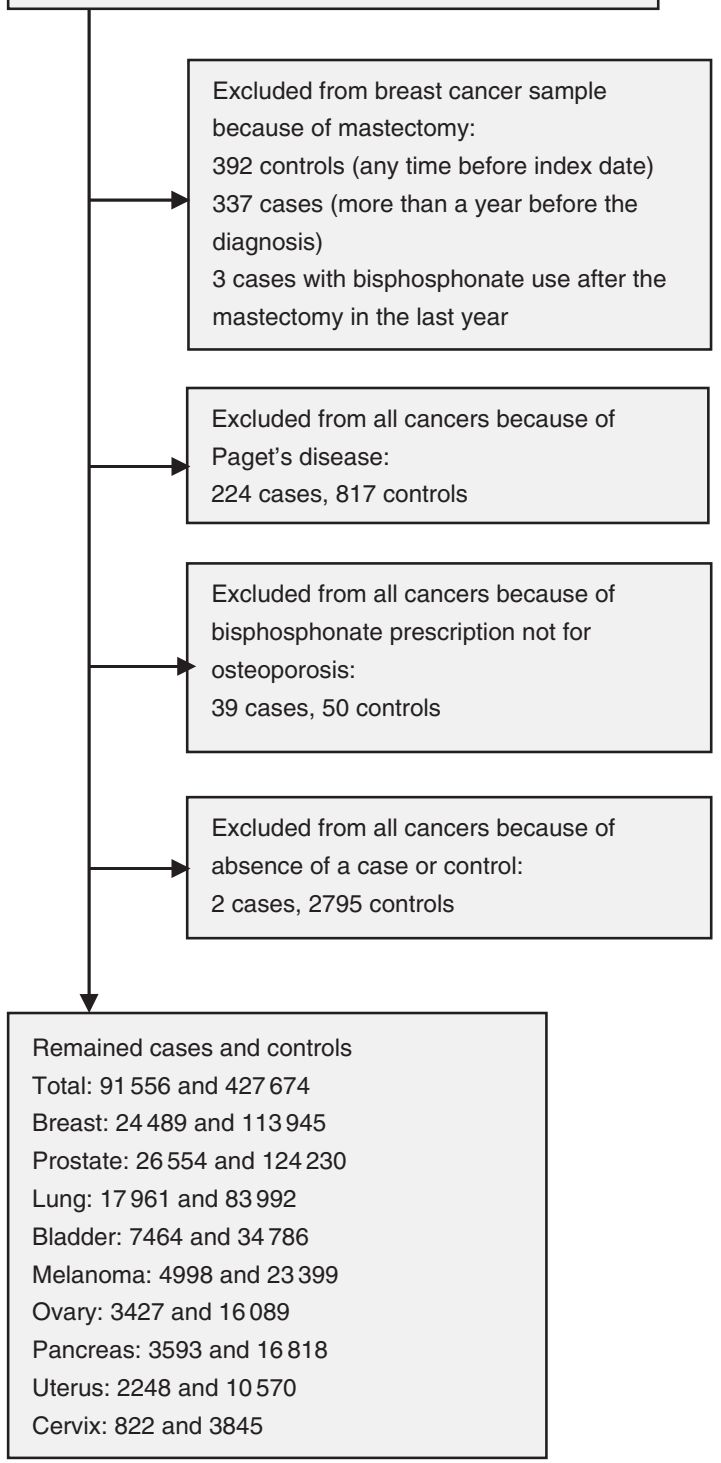

CPRD
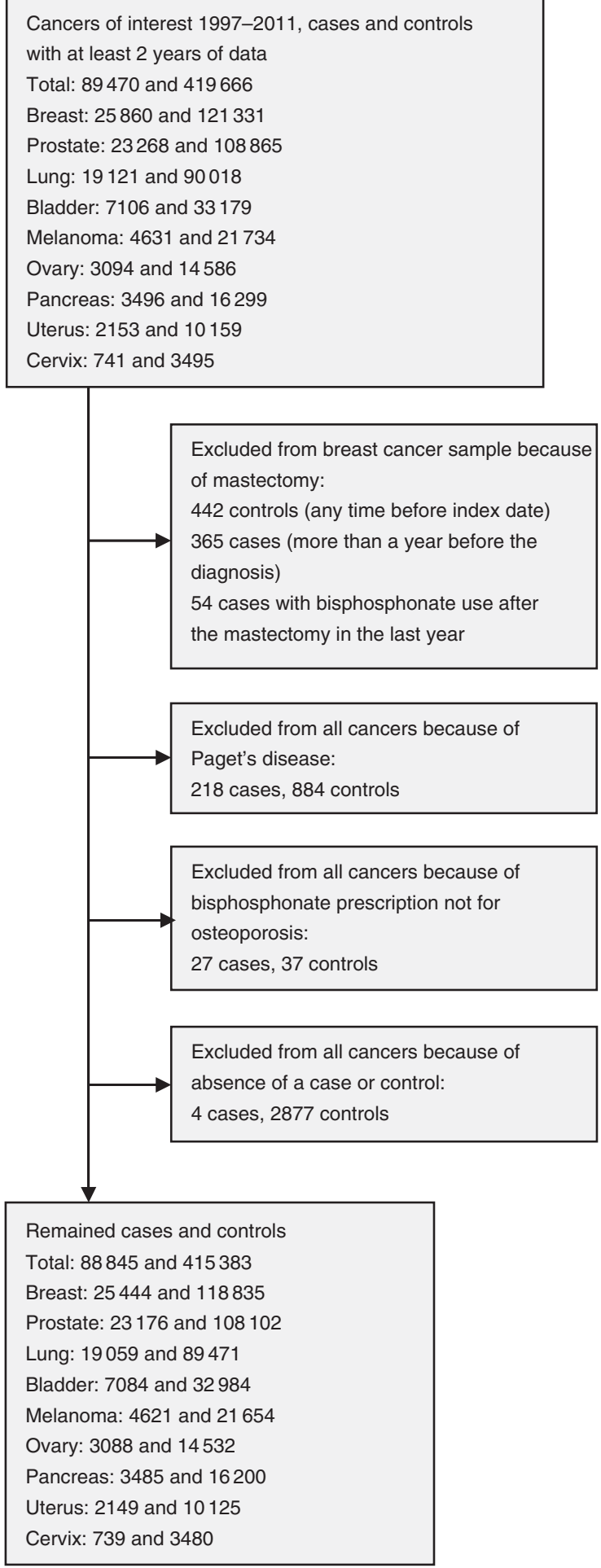

Figure 1. Flow of the included patients for QResearch and CPRD analyses.

Further analyses showed significantly reduced risks for alendronate users in QResearch, with a greater reduced risk for long-term users (AOR: 0.75; 95\% CI: 0.62-0.92), which was duration-dependent $\left(P_{\text {trend }}=0.009\right)$. Clinical Practice Research Datalink associations were directionally similar to QResearch but not significant and also with lower risk for long-term users (AOR: 0.86; 95\% CI: $0.70-1.05)$ and not duration-dependent
$\left(P_{\text {trend }}=0.05\right)$, although contributing to a significant association (AOR: 0.80; 95\% CI: 0.70-0.92) and duration dependency $\left(P_{\text {trend }}=0.001\right)$ in the combined analysis.

All sensitivity analyses with other definitions of use were consistent in showing a reduced risk in long-term alendronate users apart from the one including prescriptions from the past 6 months before the index date. This analysis also 
Table 1. Baseline characteristics in cases and all matched controls by database (QResearch or CPRD)

\begin{tabular}{|c|c|c|c|c|}
\hline & \multicolumn{2}{|c|}{ QResearch } & \multicolumn{2}{|c|}{ CPRD } \\
\hline & Cases & Controls & Cases & Controls \\
\hline Total & 91556 & 427674 & 88845 & 415383 \\
\hline $\begin{array}{l}\text { Breast } \\
\text { Prostate } \\
\text { Lung } \\
\text { Bladder } \\
\text { Skin } \\
\text { Ovary } \\
\text { Pancreas } \\
\text { Uterus } \\
\text { Cervix }\end{array}$ & $\begin{array}{c}24489 \\
26554 \\
17961 \\
7464 \\
4998 \\
3427 \\
3593 \\
2248 \\
822\end{array}$ & $\begin{array}{c}113945 \\
124230 \\
83992 \\
34786 \\
23399 \\
16089 \\
16818 \\
10570 \\
3845\end{array}$ & $\begin{array}{c}25444 \\
23176 \\
19059 \\
7084 \\
4621 \\
3088 \\
3485 \\
2149 \\
739\end{array}$ & $\begin{array}{c}118835 \\
108102 \\
89471 \\
32984 \\
21654 \\
14532 \\
16200 \\
10125 \\
3480\end{array}$ \\
\hline \multicolumn{5}{|l|}{ Sex } \\
\hline $\begin{array}{l}\text { Male } \\
\text { Female }\end{array}$ & $\begin{array}{l}47193(51.5) \\
44363(48.5)\end{array}$ & $\begin{array}{l}220492(51.6) \\
207182(48.4)\end{array}$ & $\begin{array}{l}43596(49.1) \\
45249(50.9)\end{array}$ & $\begin{array}{l}203487(49.0) \\
211896(51.0)\end{array}$ \\
\hline \multicolumn{5}{|l|}{ Age band (years) } \\
\hline $\begin{array}{l}30-54 \\
55-64 \\
65-74 \\
75-84 \\
85+\end{array}$ & $\begin{array}{c}7345(8.0) \\
22786(24.9) \\
30410(33.2) \\
26177(28.6) \\
4838(5.3)\end{array}$ & $\begin{array}{c}34000(7.9) \\
106266(24.8) \\
143528(33.6) \\
122798(28.7) \\
21082(4.9)\end{array}$ & $\begin{array}{c}6998(7.9) \\
22071(24.8) \\
28407(32.0) \\
23864(26.9) \\
7505(8.4)\end{array}$ & $\begin{array}{c}32749(7.9) \\
104095(25.1) \\
134902(32.5) \\
112533(27.1) \\
31104(7.5)\end{array}$ \\
\hline \multicolumn{5}{|l|}{ Ethnicity } \\
\hline $\begin{array}{l}\text { White } \\
\text { Not recorded } \\
\text { Non-white } \\
\text { Asian } \\
\text { Black }^{\mathbf{b}} \\
\text { Other }^{\mathrm{b}}\end{array}$ & $\begin{array}{c}21477(23.5) \\
69131(75.5) \\
948(1.0) \\
364(0.4) \\
397(0.4) \\
187(0.2)\end{array}$ & $\begin{array}{c}90874(21.2) \\
331241(77.5) \\
5559(1.3) \\
2726(0.6) \\
1834(0.4) \\
999(0.2)\end{array}$ & $\begin{array}{r}7034(7.9) \\
78841(88.7) \\
2970(3.3) \\
194(0.2) \\
166(0.2) \\
2610(2.9)\end{array}$ & $\begin{array}{r}30294(7.3) \\
371491(89.4) \\
13598(3.3) \\
1516(0.4) \\
649(0.2) \\
11433(2.8)\end{array}$ \\
\hline \multicolumn{5}{|c|}{ Deprivation, Townsend quintile } \\
\hline $\begin{array}{l}1, \text { most affluent }^{c} \\
2^{c} \\
3^{c} \\
4^{c} \\
5, \text { most deprived } \\
\text { Not recorded }\end{array}$ & $\begin{array}{l}23442(26.3) \\
19959(22.4) \\
18032(20.2) \\
15768(17.7) \\
12075(13.5) \\
2280(2.5)\end{array}$ & $\begin{array}{c}110919(26.7) \\
94167(22.7) \\
84427(20.3) \\
72202(17.4) \\
53799(12.9) \\
12160(2.8)\end{array}$ & $\begin{array}{r}9753(30.5) \\
7695(24.0) \\
6253(19.5) \\
5007(15.6) \\
3290(10.3) \\
56847(64.0)\end{array}$ & $\begin{array}{r}45736(30.6) \\
36203(24.2) \\
29740(19.9) \\
23075(15.4) \\
14914(10.0) \\
265715(64.0)\end{array}$ \\
\hline \multicolumn{5}{|l|}{ BMI $\left(\mathrm{kg} \mathrm{m}^{-2}\right)$} \\
\hline $\begin{array}{l}15-24^{c} \\
25-29^{c} \\
30-49^{c} \\
\text { Not recorded }\end{array}$ & $\begin{array}{l}27225(38.0) \\
28956(40.4) \\
15409(21.5) \\
19966(21.8)\end{array}$ & $\begin{array}{r}120028(37.3) \\
130874(40.7) \\
70904(22.0) \\
105868(24.8)\end{array}$ & $\begin{array}{l}29310(39.1) \\
29808(39.7) \\
15913(21.2) \\
13814(15.5)\end{array}$ & $\begin{array}{r}131726(38.3) \\
138713(40.3) \\
73554(21.4) \\
71390(17.2)\end{array}$ \\
\hline \multicolumn{5}{|l|}{ Smoking status } \\
\hline $\begin{array}{l}\text { Non-smoker }{ }^{c} \\
\text { Ex-smoker }^{c} \\
\text { Current light smoker } \\
\text { Current moderate smoker } \\
\text { Current heavy smoker } \\
\text { Not recorded }\end{array}$ & $\begin{array}{c}34949(41.4) \\
31475(37.3) \\
5633(6.7) \\
7825(9.3) \\
4467(5.3) \\
7207(7.9)\end{array}$ & $\begin{array}{c}182349(48.0) \\
136575(36.0) \\
20787(5.5) \\
26747(7.0) \\
13187(3.5) \\
48029(11.2)\end{array}$ & $\begin{array}{c}40128(47.6) \\
26958(32.0) \\
3966(4.7) \\
8838(10.5) \\
4382(5.2) \\
4573(5.1)\end{array}$ & $\begin{array}{c}212180(55.4) \\
112142(29.3) \\
13871(3.6) \\
30846(8.1) \\
13646(3.6) \\
32698(7.9)\end{array}$ \\
\hline \multicolumn{5}{|l|}{ Use of alcohol } \\
\hline $\begin{array}{l}\text { No use }^{c} \\
\text { Ex use }^{c} \\
\text { Light }^{c} \\
\text { Moderate and more } \\
\text { Not recorded }\end{array}$ & $\begin{array}{c}22061(29.9) \\
44809(60.7) \\
7009(9.5) \\
17677(19.3)\end{array}$ & $\begin{array}{c}101997(30.5) \\
203342(60.8) \\
29036(8.7) \\
93299(21.8)\end{array}$ & $\begin{array}{c}19714(25.8) \\
2030(2.7) \\
44448(58.1) \\
10250(13.4) \\
12403(14.0)\end{array}$ & $\begin{array}{c}92647(26.6) \\
7672(2.2) \\
204556(58.8) \\
42911(12.3) \\
67597(16.3)\end{array}$ \\
\hline
\end{tabular}




\section{Table 1. (Continued)}

\begin{tabular}{|c|c|c|c|c|}
\hline & \multicolumn{2}{|c|}{ QResearch } & \multicolumn{2}{|c|}{ CPRD } \\
\hline & Cases & Controls & Cases & Controls \\
\hline \multicolumn{5}{|l|}{ Comorbidities } \\
\hline $\begin{array}{l}\text { Upper Gl } \\
\text { Diabetes } \\
\text { Pancreatitis } \\
\text { Hypertension } \\
\text { Chronic kidney disease } \\
\text { Rheumatoid arthritis } \\
\text { Benign breast disease }\end{array}$ & $\begin{array}{c}19250(21.0) \\
8140(8.9) \\
492(0.5) \\
31466(34.4) \\
3444(3.8) \\
1424(1.6) \\
2727(3.0)\end{array}$ & $\begin{array}{c}83377(19.5) \\
36769(8.6) \\
1981(0.5) \\
142577(33.3) \\
15558(3.6) \\
6591(1.5) \\
10077(2.4) \\
\end{array}$ & $\begin{array}{c}24302(27.4) \\
7990(9.0) \\
552(0.6) \\
31169(35.1) \\
3710(4.2) \\
1673(1.9) \\
2761(3.1)\end{array}$ & $\begin{array}{r}107660(25.9) \\
37011(8.9) \\
2102(0.5) \\
142474(34.3) \\
16425(4.0) \\
7310(1.8) \\
9653(2.3) \\
\end{array}$ \\
\hline \multicolumn{5}{|l|}{ History of osteoporosis } \\
\hline $\begin{array}{l}\text { Osteoporosis/osteopenia } \\
\text { Osteoporotic fractures }\end{array}$ & $\begin{array}{l}3682(4.0) \\
3322(3.6)\end{array}$ & $\begin{array}{l}16998(4.0) \\
16013(3.7)\end{array}$ & $\begin{array}{l}4023(4.5) \\
2375(2.7)\end{array}$ & $\begin{array}{l}18474(4.4) \\
10342(2.5)\end{array}$ \\
\hline Family history of cancer & $2841(3.1)$ & $11756(2.7)$ & $2836(3.2)$ & $11364(2.7)$ \\
\hline \multicolumn{5}{|c|}{ Medications (excluding past 6 months) } \\
\hline $\begin{array}{l}\text { Acid-lowering drugs } \\
\text { NSAIDs } \\
\text { Corticosteroids } \\
\text { Calcium } \\
\text { Vitamin D } \\
\text { Hormone replacement }^{\text {d }} \\
\text { Oral contraceptives }^{d}\end{array}$ & $\begin{array}{c}32523(35.5) \\
52422(57.3) \\
14156(15.5) \\
1023(1.1) \\
4891(5.3) \\
12071(13.2) \\
773(0.8)\end{array}$ & $\begin{array}{c}140637(32.9) \\
233143(54.5) \\
57106(13.4) \\
4816(1.1) \\
22582(5.3) \\
51861(12.1) \\
3551(0.8) \\
\end{array}$ & $\begin{array}{c}33531(37.7) \\
55106(62.0) \\
13908(15.7) \\
6286(7.1) \\
4960(5.6) \\
12633(14.2) \\
1236(1.4)\end{array}$ & $\begin{array}{c}145409(35.0) \\
248518(59.8) \\
54233(13.1) \\
28984(7.0) \\
23035(5.5) \\
55666(13.4) \\
5490(1.3) \\
\end{array}$ \\
\hline \multicolumn{5}{|l|}{ Bisphosphonates } \\
\hline $\begin{array}{l}\text { Any } \\
\text { Alendronate } \\
\text { Etidronate } \\
\text { Risedronate } \\
\text { Ibandronate }\end{array}$ & $\begin{array}{r}3827(4.2) \\
2589(2.8) \\
1286(1.4) \\
851(0.9) \\
74(0.1)\end{array}$ & $\begin{array}{r}17883(4.2) \\
12010(2.8) \\
5949(1.4) \\
3985(0.9) \\
435(0.1)\end{array}$ & $\begin{array}{r}3769(4.2) \\
2538(2.9) \\
1170(1.3) \\
806(0.9) \\
75(0.1)\end{array}$ & $\begin{array}{r}17490(4.2) \\
11969(2.9) \\
5471(1.3) \\
3715(0.9) \\
353(0.1)\end{array}$ \\
\hline \multicolumn{5}{|c|}{ Other osteoporosis drugs } \\
\hline $\begin{array}{l}\text { Any } \\
\text { Raloxifen } \\
\text { Strontium } \\
\text { Calcitonin }\end{array}$ & $\begin{array}{r}319(0.3) \\
192(0.2) \\
95(0.1) \\
38(0.0)\end{array}$ & $\begin{array}{r}1641(0.4) \\
1032(0.2) \\
456(0.1) \\
207(0.0)\end{array}$ & $\begin{array}{r}333(0.4) \\
217(0.2) \\
97(0.1) \\
34(0.0)\end{array}$ & $\begin{array}{r}1685(0.4) \\
1081(0.3) \\
509(0.1) \\
162(0.0)\end{array}$ \\
\hline \multicolumn{5}{|c|}{$\begin{array}{l}\text { Abbreviations: CPRD = Clinical Practice Research Datalink; GI = gastrointestinal; NSAID = non-steroidal anti-inflammatory drugs. Values are numbers and \%. } \\
\text { a Assumed White for the analyses. } \\
\text { b Broken down categories for non-White group. } \\
\text { P Proportion only within recorded data. } \\
\text { dOnly for women cancers. }\end{array}$} \\
\hline
\end{tabular}

demonstrated an increased risk for very short-term use (up to 6 months) but only in QResearch (AOR: 1.29; 95\% CI: 1.08-1.53, $P=0.004)$. As for overall bisphosphonate use, the sensitivity analysis for at least two prescriptions reached a statistically significant level in CPRD (AOR: 0.83, 95\% CI: 0.72-0.95, $P=0.008)$.

Lung cancer. After adjusting for confounders, there was no significant association with bisphosphonate use (AOR: 0.97, 95\% CI: $0.88-1.08$ in QResearch; 1.12, 1.01-1.23 in CPRD; 1.04, 0.971.12 for combined). Further analyses demonstrated no associations with duration or type of bisphosphonate.

Results were consistent in sensitivity analyses apart from one - the sensitivity analysis, which included prescriptions from the past 6 months before the index date showed increased risk associated with short-term use in both databases (AOR: 1.34, 95\%
CI: $1.18-1.52, P<0.001$ for QResearch; 1.30, 1.15-1.47, $P<0.001$ for CPRD).

Bladder cancer. Adjusting for confounders showed no association between bisphosphonate use and risk of bladder cancer (AOR: 0.96, 95\% CI: $0.80-1.14$ for QResearch; 0.94, 0.79-1.12 for CPRD; $0.95,0.84-1.08$ for combined). Sensitivity analyses also showed no associations.

Melanoma. There was no association between overall bisphosphonate use and risk of melanoma in either database (AOR: 1.05, 95\% CI: 0.87-1.28 for QResearch; 0.95, 0.77-1.19 for CPRD; $1.01,0.87-1.17$ for combined), and analyses did not show statistically significantly increased risk with longer use in either database or the combined $\left(P_{\text {trend }}=0.013\right.$ for QResearch; $P_{\text {trend }}=0.5$ for CPRD; $P_{\text {trend }}=0.02$ for combined). The results were consistent across the sensitivity analyses, except for the one defining use as at least one prescription between 6 and 72 months. 


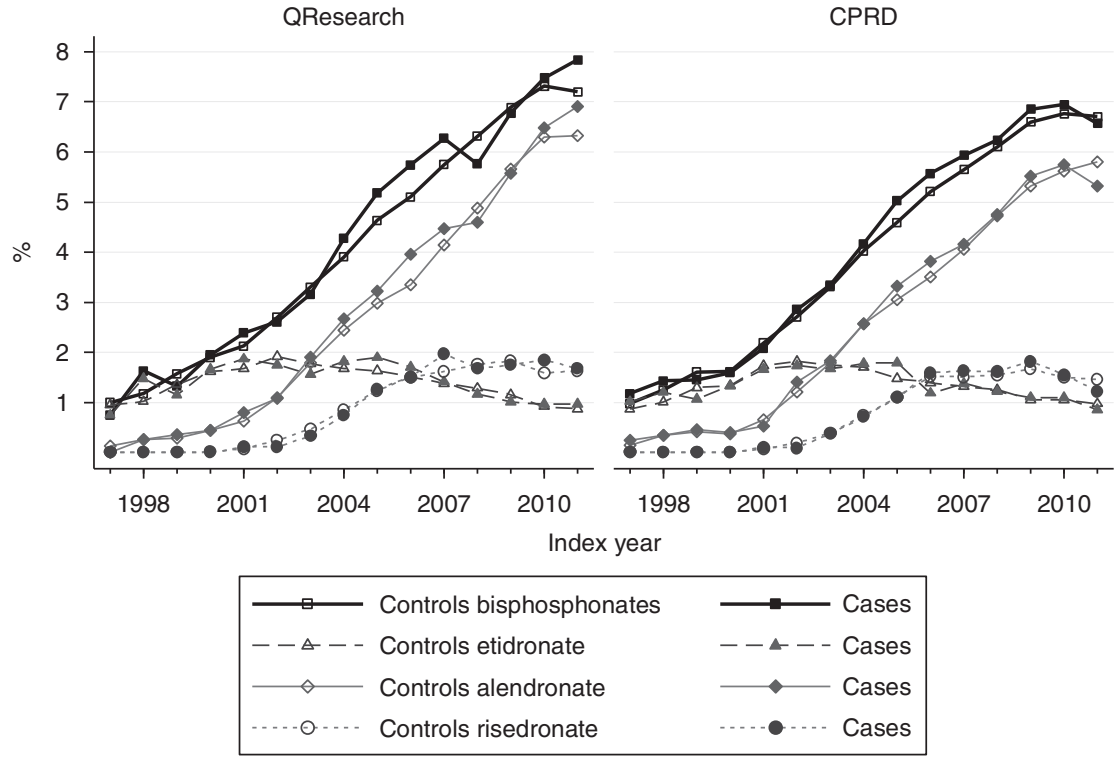

() QRESEARCH 2011 version 31, CPRD March 2012

Figure 2. Proportion of cases and controls in QResearch and CPRD with at least one prescription of bisphosphonate by index year.

\begin{tabular}{|c|c|c|c|c|c|c|c|c|}
\hline \multirow[b]{2}{*}{ Cancer site } & \multicolumn{3}{|c|}{ QResearch } & \multicolumn{3}{|c|}{ CPRD } & \multicolumn{2}{|c|}{ Combined analysis } \\
\hline & $\begin{array}{l}\text { Cases/ } \\
\text { controls }\end{array}$ & $\begin{array}{l}\text { Adjusted }{ }^{*} \text { odds } \\
\text { ratio }(95 \% \mathrm{Cl})\end{array}$ & $\boldsymbol{P}$-value & $\begin{array}{l}\text { Cases/ } \\
\text { controls }\end{array}$ & $\begin{array}{l}\text { Adjusted"odds } \\
\text { ratio }(95 \% \mathrm{Cl})\end{array}$ & $\boldsymbol{P}$-value & $\begin{array}{l}\text { Pooled odds } \\
\text { ratio }(95 \% \mathrm{Cl})\end{array}$ & $P$-value \\
\hline Breast $^{a}$ & $1304 / 6923$ & $0.89(0.82-0.97)$ & 0.005 & $1324 / 6847$ & $0.95(0.88-1.03)$ & 0.2 & $0.92(0.87-0.97)$ & 0.004 \\
\hline Prostate $^{\mathbf{b}}$ & $460 / 2150$ & $0.90(0.79-1.02)$ & 0.1 & $376 / 1901$ & $0.84(0.73-0.96)$ & 0.012 & $0.87(0.79-0.96)$ & 0.003 \\
\hline Lung $^{c}$ & $1035 / 3809$ & $0.97(0.88-1.08)$ & 0.6 & $1114 / 3911$ & $1.12(1.01-1.23)$ & 0.03 & $1.04(0.97-1.12)$ & 0.2 \\
\hline Bladder $^{\text {d }}$ & $274 / 1219$ & $0.96(0.80-1.14)$ & 0.6 & $280 / 1263$ & $0.94(0.79-1.12)$ & 0.5 & $0.95(0.84-1.08)$ & 0.4 \\
\hline Melanoma ${ }^{\mathbf{e}}$ & $241 / 1067$ & $1.05(0.87-1.28)$ & 0.6 & $178 / 881$ & $0.95(0.77-1.19)$ & 0.7 & $1.01(0.87-1.17)$ & 0.9 \\
\hline Ovary $^{f}$ & $204 / 939$ & $1.19(0.96-1.47)$ & 0.1 & $170 / 937$ & $0.84(0.67-1.04)$ & 0.1 & $1.00(0.86-1.16)$ & 1.0 \\
\hline Pancreas $^{9}$ & $178 / 886$ & $0.81(0.64-1.01)$ & 0.06 & $196 / 918$ & $0.78(0.63-0.97)$ & 0.03 & $0.79(0.68-0.93)$ & 0.003 \\
\hline Uterus $^{h}$ & $99 / 671$ & $1.07(0.79-1.44)$ & 0.7 & $96 / 636$ & $0.95(0.71-1.27)$ & 0.7 & $1.00(0.81-1.24)$ & 1.0 \\
\hline Cervix $^{i}$ & $32 / 219$ & $0.78(0.48-1.27)$ & 0.3 & $35 / 196$ & $1.21(0.76-1.93)$ & 0.4 & $0.98(0.70-1.37)$ & 0.9 \\
\hline 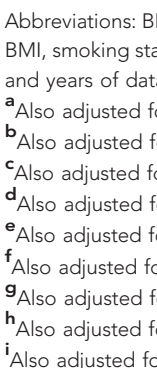 & $\begin{array}{l}\text { ody mass index; } \\
\text { cohol consumpt } \\
\text { ily history of bre } \\
\text { ily history of pro } \\
\text { ily history of lun } \\
\text { ily history of can } \\
\text { ily history of can } \\
\text { ly history of ova } \\
\text { ily history of Gl } \\
\text { ily history of can } \\
\text { ly history of can }\end{array}$ & $\begin{array}{l}\text { confidence interval; CPR } \\
\text { thnicity, rheumatoid artt } \\
\text { ancer, benign breast dis } \\
\text { cancer. } \\
\text { cer. } \\
\text { and chronic kidney disea } \\
\text { ancer, use of oral contr } \\
\text { er, Gl disease, diabetes } \\
\text { hypertension, diabetes, } \\
\text { se of oral contraceptive }\end{array}$ & $\begin{array}{l}\text { ives and } h \\
\text { istory of } p \\
\text { f oral cont } \\
\text { hormone }\end{array}$ & $\begin{array}{l}\text { Research Datali } \\
\text { d fractures, use } \\
\text { raceptives and }\end{array}$ & $\begin{array}{l}\mathrm{Gl}=\text { gastrointestinal; } \\
\text { other osteoporosis dr } \\
\text { mone replacement th }\end{array}$ & non & $\begin{array}{l}\text { inflammatory dru } \\
\text { rticosteroids, aci }\end{array}$ & $\begin{array}{l}\text { djusted } f \\
\text { ring drus }\end{array}$ \\
\hline
\end{tabular}

Here use of etidronate in the QResearch analysis was associated with increased risk of melanoma (AOR: 1.67, 95\% CI: 1.23-2.26, $P<0.001)$; however, this association was not duration-dependent $\left(P_{\text {trend }}=0.02\right)$.

Pancreatic cancer. After adjusting for the confounders analyses in both databases showed a reduced risk of pancreatic cancer in bisphosphonate users, although it was not statistically significant (AOR: 0.81, 95\% CI: 0.64-1.01 for QResearch; 0.78, 0.63-0.97 for CPRD). The combined analysis, however, demonstrated a statistically significant association (AOR: 0.79, 95\% CI: 0.68-0.93). Short-term use was associated with an even lower but not statistically significant risk in all analyses (AOR: 0.78, 95\% CI: 0.57-1.06 for QResearch; 0.77, 0.57-1.02 for CPRD; 0.77, 


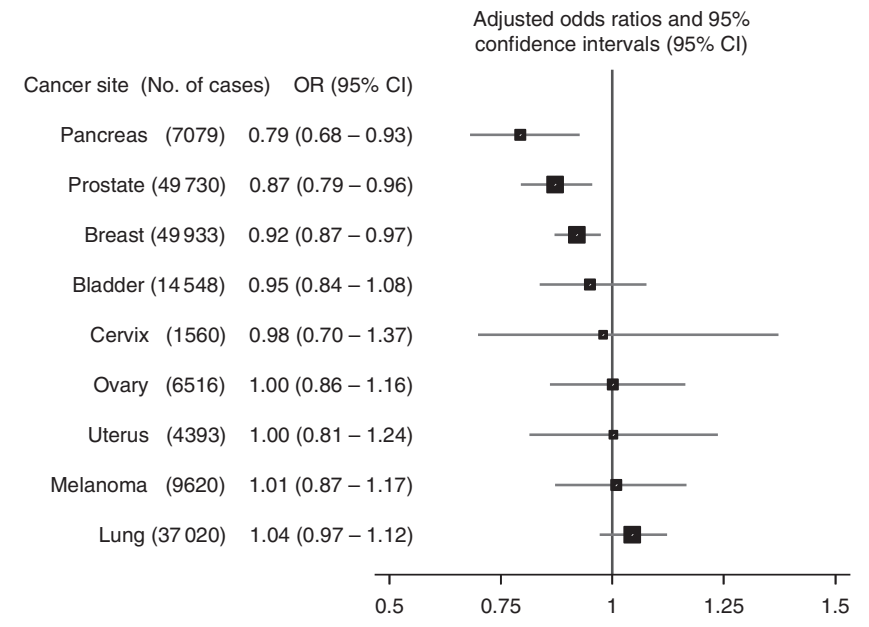

Odds ratios and $95 \% \mathrm{Cl}$ are adjusted for deprivation, smoking, comorbidities, use of medication

Reference group: No prescriptions of bisphosphonates

(c) QRESEARCH 2011 version 31, CPRD March 2012

Figure 3. Risk of cancer in patients prescribed bisphosphonates, combined results for QResearch and CPRD analyses.

0.63-0.96 for combined) and there was also no trend for duration of use.

A sensitivity analysis defining use as at least two prescriptions reached statistically significant level for the CPRD analysis in overall use $(0.73,0.59-0.91, P=0.005)$ and for short-term use (AOR: $0.65,95 \% \mathrm{CI}: 0.47-0.89, P=0.008$ ). The other sensitivity analyses did not demonstrate any significant associations.

Additional information. Sensitivity analyses on patients with valid BMI, smoking status and alcohol consumption data and on patients with valid Townsend codes showed similar results, which are available from the authors.

\section{DISCUSSION}

Summary. This study, based on medical records from the two largest UK primary-care databases, showed that the use of bisphosphonates was not associated with increased risks for any of the most common solid non-gastrointestinal cancers. Decreased risks of breast, prostate and pancreatic cancers had no duration relationship with bisphosphonate use and were found only in short-term users. Alendronate use, however, was associated with a decreased risk of prostate cancer and this association was durationdependent.

Strengths and limitations. Based on the two largest primary-care databases, including more recent data than previous studies and covering the period when bisphosphonates have become much more widely prescribed in the general population, our study provides more data for investigating the duration effects of bisphosphonate use and over longer periods than previous studies. All eligible cancer cases and controls, alive or dead, were included into the study. This representativeness of the databases, and the lack of recall, selection and respondent biases, also makes the results more generalisable.

The limitations include possible uncertainties in cancer diagnosis. The selection of cases was based on the first record of a cancer and the exact origin site may have been determined only later, but this level of detail was not available across all records. A systematic review based on GPRD validation studies reported that, on average, $95 \%$ of diagnoses of cancer recorded on the GP electronic record were confirmed by other data sources (Herrett et al, 2010). Information about cancer stage and the results of histological investigations were also not consistently recorded across general practices and so were not used. Another limitation is the potential misclassification of bisphosphonate use. The analyses were based on prescriptions not on actual use and no data were available on adherence to medications. There is no reason, however, to suppose that non-adherence differs systematically between cases and controls.

Other limitations include information bias and missing data. Lack of symptom or family history records might arise simply because the patients have not reported them. Information on certain risk factors such as the level of physical activity, diet and cancer screening tests (mammography, prostate-specific antigen test) was also not reliably recorded, so these factors were not included in the analyses. Further, results of any bone mineral density tests were not consistently recorded and were not used in the analysis, and so there might be some residual confounding. Important confounders such as smoking or BMI had some missing data, so these had to be imputed.

Bisphosphonate users. Comparisons between bisphosphonate users and non-users reflect the recommendations for targeting the group with osteoporosis, and so some characteristics and comorbidities such as low BMI and rheumatoid arthritis in users were expected as these are risk factors for primary osteoporosis. As secondary osteoporosis is more likely to develop in patients with impaired digestion, use of acid-lowering drugs and also use of corticosteroids (Mauck and Clarke, 2006; Hippisley-Cox and Coupland, 2009) were much higher for bisphosphonate users.

Breast cancer. Reduced breast cancer risk associated with bisphosphonates use has been shown in five studies to date, all smaller than the current one (Chlebowski et al, 2010; Newcomb et al, 2010; Rennert et al, 2010; Vestergaard et al, 2011; Cardwell et al, 2012). No relation between risk and duration of use could suggest that osteoporosis is responsible for such reductions (Chen et al, 2008). Adjustments for osteoporosis, however, have demonstrated the independent effect of bisphosphonates. Reduced risk associated with recent, but not remote, use of bisphosphonates might also be explained by a possible bisphosphonate-related prevention of progress for undiagnosed cancers up to an invasive stage. This has already been shown by Chlebowski's study (Chlebowski et al, 2010) and supported by studies in menopausal women with early-stage breast cancer treated with bisphosphonates (Gnant, 2010). Although an Israeli (Rennert et al, 2010) case-control study showed a statistically significantly decreased risk associated with more than a year of bisphosphonate use (AOR: 0.61, 95\% CI: 0.500.76 ), the study was subject to recall bias (participants reported their past use of bisphosphonates) and selection bias (all participants were alive at the moment of the interview). Another case-control study (Newcomb et al, 2010) with similar limitations in the design also reported a statistically significant trend $\left(P_{\text {trend }}=0.01\right)$ for duration of bisphosphonate use. A cohort study of Cardwell et al (2012) showed risk reduction associated with overall bisphosphonate use similar to ours (adjusted hazard ratio (AHR): 0.79 , 95\% CI: 0.62-1.01), and also becoming not statistically significant for patients with use of more than a year. Although our results for different types of bisphosphonate were in line with Vestergaard et al's study (2011) for alendronate (AHR: 0.91, 95\% CI: $0.81-1.03$ ), the risk reduction for etidronate in our study did not reach a statistically significant level.

Prostate cancer. The association between overall use of bisphosphonates and prostate cancer risk was similar to that for breast cancer, also without statistically significant effects for therapy duration. Although the association between alendronate use and 
Table 3. Bisphosphonate short- and long-term use in cancer cases and controls, numbers and odds ratios (95\% Cls) compared with non-use by database

\begin{tabular}{|c|c|c|c|c|c|c|c|c|}
\hline \multirow{2}{*}{$\begin{array}{l}\text { Cancer site } \\
\text { Terms of use }\end{array}$} & \multicolumn{3}{|c|}{ QResearch } & \multicolumn{3}{|c|}{ CPRD } & \multicolumn{2}{|c|}{ Combined analysis } \\
\hline & $\begin{array}{l}\text { Cases/ } \\
\text { controls }\end{array}$ & $\begin{array}{l}\text { Adjusted }{ }^{*} \text { odds } \\
\text { ratio }(95 \% \mathrm{Cl})\end{array}$ & $\boldsymbol{P}$-value & $\begin{array}{l}\text { Cases/ } \\
\text { controls }\end{array}$ & $\begin{array}{l}\text { Adjusted }{ }^{*} \text { odds } \\
\text { ratio }(95 \% \mathrm{Cl})\end{array}$ & $\boldsymbol{P}$-value & $\begin{array}{l}\text { Pooled odds } \\
\text { ratio }(95 \% \mathrm{Cl})\end{array}$ & $\boldsymbol{P}$-value \\
\hline Breast & Trend & & 0.5 & Trend & & 0.3 & Trend & 0.2 \\
\hline $\begin{array}{l}\text { Short term } \\
\text { (less than a year) } \\
\text { Long term } \\
\text { (at least a year) }\end{array}$ & $\begin{array}{l}462 / 2523 \\
842 / 4400\end{array}$ & $\begin{array}{l}0.86(0.77-0.97) \\
0.91(0.83-1.00)\end{array}$ & $\begin{array}{l}0.01 \\
0.05\end{array}$ & $\begin{array}{l}508 / 2620 \\
816 / 4227\end{array}$ & $\begin{array}{l}0.95(0.86-1.06) \\
0.95(0.86-1.04)\end{array}$ & $\begin{array}{l}0.4 \\
0.3\end{array}$ & $\begin{array}{l}0.91(0.84-0.98) \\
0.93(0.87-0.99)\end{array}$ & $\begin{array}{l}0.02 \\
0.03\end{array}$ \\
\hline Prostate & Trend & & 0.06 & Trend & & 0.03 & Trend & 0.005 \\
\hline $\begin{array}{l}\text { Short term } \\
\text { (less than a year) } \\
\text { Long term } \\
\text { (at least a year) }\end{array}$ & $\begin{array}{c}186 / 838 \\
274 / 1312\end{array}$ & $\begin{array}{l}0.95(0.80-1.13) \\
0.86(0.74-1.00)\end{array}$ & $\begin{array}{l}0.6 \\
0.06\end{array}$ & $\begin{array}{l}149 / 764 \\
227 / 1137\end{array}$ & $\begin{array}{l}0.84(0.70-1.02) \\
0.84(0.71-0.99)\end{array}$ & $\begin{array}{l}0.07 \\
0.04\end{array}$ & $\begin{array}{l}0.90(0.79-1.02) \\
0.85(0.76-0.95)\end{array}$ & $\begin{array}{l}0.1 \\
0.005\end{array}$ \\
\hline Lung & Trend & & 0.7 & Trend & & 1.0 & Trend & 0.8 \\
\hline $\begin{array}{l}\text { Short term } \\
\text { (less than a year) } \\
\text { Long term } \\
\text { (at least a year) }\end{array}$ & $\begin{array}{l}415 / 1351 \\
620 / 2458\end{array}$ & $\begin{array}{l}1.04(0.90-1.19) \\
0.93(0.82-1.05)\end{array}$ & $\begin{array}{l}0.6 \\
0.2\end{array}$ & $\begin{array}{l}467 / 1507 \\
647 / 2404\end{array}$ & $\begin{array}{l}1.15(1.01-1.32) \\
1.09(0.96-1.23)\end{array}$ & $\begin{array}{l}0.03 \\
0.2\end{array}$ & $\begin{array}{l}1.10(1.00-1.21) \\
1.01(0.92-1.10)\end{array}$ & $\begin{array}{l}0.06 \\
0.9\end{array}$ \\
\hline Bladder & Trend & & 0.3 & Trend & & 0.6 & Trend & 0.3 \\
\hline $\begin{array}{l}\text { Short term } \\
\text { (less than a year) } \\
\text { Long term } \\
\text { (at least a year) }\end{array}$ & $\begin{array}{l}109 / 455 \\
165 / 764\end{array}$ & $\begin{array}{l}0.99(0.79-1.26) \\
0.93(0.76-1.15)\end{array}$ & $\begin{array}{l}1.0 \\
0.5\end{array}$ & $\begin{array}{l}117 / 464 \\
163 / 799\end{array}$ & $\begin{array}{l}1.06(0.84-1.33) \\
0.86(0.69-1.06)\end{array}$ & $\begin{array}{l}0.6 \\
0.2\end{array}$ & $\begin{array}{l}1.03(0.87-1.21) \\
0.90(0.77-1.04)\end{array}$ & $\begin{array}{l}0.8 \\
0.2\end{array}$ \\
\hline Melanoma & Trend & & 0.01 & Trend & & 0.5 & Trend & 0.02 \\
\hline $\begin{array}{l}\text { Short term } \\
\text { (less than a year) } \\
\text { Long term } \\
\text { (at least a year) }\end{array}$ & $\begin{array}{l}77 / 400 \\
164 / 667\end{array}$ & $\begin{array}{l}0.91(0.70-1.20) \\
1.16(0.92-1.45)\end{array}$ & $\begin{array}{l}0.5 \\
0.2\end{array}$ & $\begin{array}{l}64 / 354 \\
114 / 527\end{array}$ & $\begin{array}{l}0.88(0.65-1.18) \\
1.01(0.78-1.31)\end{array}$ & $\begin{array}{l}0.4 \\
0.9\end{array}$ & $\begin{array}{l}0.90(0.73-1.10) \\
1.09(0.92-1.29)\end{array}$ & $\begin{array}{l}0.3 \\
0.3\end{array}$ \\
\hline Ovaries & Trend & & 0.4 & Trend & & 0.1 & Trend & 0.7 \\
\hline $\begin{array}{l}\text { Short term } \\
\text { (less than a year) } \\
\text { Long term } \\
\text { (at least a year) }\end{array}$ & $\begin{array}{l}83 / 322 \\
121 / 617\end{array}$ & $\begin{array}{l}1.37(1.04-1.81) \\
1.08(0.84-1.38)\end{array}$ & $\begin{array}{l}0.02 \\
0.6\end{array}$ & $\begin{array}{l}62 / 355 \\
108 / 582\end{array}$ & $\begin{array}{l}0.80(0.59-1.08) \\
0.86(0.66-1.11)\end{array}$ & $\begin{array}{l}0.1 \\
0.2\end{array}$ & $\begin{array}{l}1.07(0.87-1.31) \\
0.96(0.81-1.15)\end{array}$ & $\begin{array}{l}0.5 \\
0.7\end{array}$ \\
\hline Pancreas & Trend & & 0.2 & Trend & & 0.05 & Trend & 0.02 \\
\hline $\begin{array}{l}\text { Short term } \\
\text { (less than a year) }\end{array}$ & $63 / 316$ & $0.78(0.57-1.06)$ & 0.1 & $71 / 336$ & $0.77(0.57-1.02)$ & 0.07 & $0.77(0.63-0.96)$ & 0.02 \\
\hline $\begin{array}{l}\text { Long term } \\
\text { (at least a year) }\end{array}$ & $115 / 570$ & $0.82(0.63-1.06)$ & 0.1 & $125 / 582$ & $0.80(0.62-1.02)$ & 0.08 & $0.81(0.67-0.97)$ & 0.02 \\
\hline
\end{tabular}

decreased risk appeared to be significant even with a duration relationship, it was found only in the QResearch database and might again suggest a possible suppressing effect of alendronate on an already existing in situ cancer (Tuomela et al, 2008). In particular in the prostate, in vitro studies have observed substantial concentrations of nitrogen-containing bisphosphonates (zolendronic acid and ibandronate) after administration of the drug (Fournier et al, 2002), which could result in suppressed angiogenesis in prostate tumours. The only epidemiological study looking at prostate cancer with respect to bisphosphonate use has also demonstrated lower risk for bisphosphonate users, although not statistically significant (AHR: 0.71, 95\% CI: 0.50-1.01) (Cardwell et al, 2012).

Lung cancer. Our study showed no association between lung cancer risk and bisphosphonate use. This is consistent with the only other epidemiological study (Cardwell et al, 2012), which also found a reduced risk for long-term users (AHR: 0.86, 95\% CI: $0.63-1.17)$ but it was not statistically significant. The antitumour properties of bisphosphonates might not be applicable in the lung because it is not a site where bisphosphonates accumulate (Fournier et al, 2002). The sensitivity analysis for all prescriptions including the past 6 months showed a significantly increased risk but without any duration relationship and in particular for shortterm users. This may simply suggest that a common symptom of lung cancer, bone pain, is sometimes initially mistaken as osteoporosis.

Pancreas. Although none of the database analyses showed statistically significantly decreased risks associated with bisphosphonate use, the combined analysis did. The findings were consistent with the only other epidemiological study (Cardwell et al, 2012) for this cancer site (AHR: 0.84, 95\% CI: 0.53-1.35). The effects of bisphosphonates on growth and apoptosis in 
Table 4. Bisphosphonate use for individual drugs in cancer cases and controls, numbers and odds ratios (95\% Cls) compared with non-use by database

QResearch

CPRD

Combined analysis

\begin{tabular}{|c|c|c|c|c|c|c|c|c|}
\hline & $\begin{array}{l}\text { Cases/ } \\
\text { controls }\end{array}$ & $\begin{array}{l}\text { Adjusted }{ }^{*} \text { odds } \\
\text { ratio }(95 \% \mathrm{Cl})\end{array}$ & $\boldsymbol{P}$-value & $\begin{array}{l}\text { Cases/ } \\
\text { controls }\end{array}$ & $\begin{array}{l}\text { Adjusted odds } \\
\text { ratio }(95 \% \mathrm{Cl})\end{array}$ & $\boldsymbol{P}$-value & $\begin{array}{l}\text { Pooled odds } \\
\text { ratio }(95 \% \mathrm{Cl})\end{array}$ & $\boldsymbol{P}$-value \\
\hline Breast, alendronate & Trend & & 0.7 & Trend & & 0.04 & Trend & 0.3 \\
\hline Any use & $901 / 4627$ & $0.98(0.89-1.07)$ & 0.6 & $892 / 4648$ & $0.96(0.88-1.05)$ & 0.3 & $0.97(0.91-1.03)$ & 0.3 \\
\hline $\begin{array}{l}\text { Short term } \\
\text { (less than a year) }\end{array}$ & $409 / 2148$ & $0.95(0.85-1.08)$ & 0.4 & $414 / 2085$ & $1.00(0.89-1.13)$ & 0.9 & $0.98(0.90-1.06)$ & 0.6 \\
\hline Long term (at least a year) & $492 / 2479$ & $1.00(0.89-1.12)$ & 1.0 & $478 / 2563$ & $0.92(0.83-1.03)$ & 0.2 & $0.96(0.89-1.04)$ & 0.3 \\
\hline Breast, etidronate & Trend & & 0.1 & Trend & & 0.8 & Trend & 0.2 \\
\hline Any use & $453 / 2437$ & $0.90(0.81-1.01)$ & 0.07 & $412 / 2246$ & $0.91(0.81-1.02)$ & 0.1 & $0.91(0.84-0.98)$ & 0.02 \\
\hline $\begin{array}{l}\text { Short term } \\
\text { (less than a year) }\end{array}$ & $176 / 893$ & $0.95(0.80-1.13)$ & 0.6 & $179 / 1018$ & $0.88(0.74-1.03)$ & 0.1 & $0.91(0.81-1.03)$ & 0.1 \\
\hline Long term (at least a year) & $277 / 1544$ & $0.87(0.76-1.00)$ & 0.05 & $233 / 1228$ & $0.94(0.81-1.09)$ & 0.4 & $0.90(0.82-1.00)$ & 0.04 \\
\hline Breast, risedronate & Trend & & 0.9 & Trend & & 0.3 & Trend & 0.5 \\
\hline Any use & $281 / 1537$ & $0.92(0.80-1.06)$ & 0.2 & $279 / 1492$ & $0.96(0.84-1.11)$ & 0.6 & $0.94(0.85-1.04)$ & 0.2 \\
\hline $\begin{array}{l}\text { Short term } \\
\text { (less than a year) }\end{array}$ & $139 / 803$ & $0.89(0.73-1.07)$ & 0.2 & $118 / 733$ & $0.83(0.68-1.02)$ & 0.07 & $0.86(0.75-0.99)$ & 0.03 \\
\hline Long term (at least a year) & $142 / 734$ & $0.96(0.80-1.16)$ & 0.7 & $161 / 759$ & $1.08(0.90-1.29)$ & 0.4 & $1.02(0.90-1.17)$ & 0.7 \\
\hline Prostate, alendronate & Trend & & 0.009 & Trend & & 0.05 & Trend & 0.001 \\
\hline Any use & $292 / 1506$ & $0.81(0.70-0.93)$ & 0.004 & $257 / 1299$ & $0.88(0.75-1.03)$ & 0.1 & $0.84(0.75-0.93)$ & 0.001 \\
\hline $\begin{array}{l}\text { Short term } \\
\text { (less than a year) }\end{array}$ & $142 / 692$ & $0.86(0.71-1.05)$ & 0.1 & $120 / 591$ & $0.91(0.74-1.13)$ & 0.4 & $0.89(0.77-1.02)$ & 0.1 \\
\hline Long term (at least a year) & $150 / 814$ & $0.75(0.62-0.92)$ & 0.004 & $137 / 708$ & $0.86(0.70-1.05)$ & 0.1 & $0.80(0.70-0.92)$ & 0.002 \\
\hline Prostate, etidronate & Trend & & 0.9 & Trend & & 0.6 & Trend & 0.8 \\
\hline any use & $141 / 567$ & $1.06(0.87-1.30)$ & 0.5 & $104 / 496$ & $0.88(0.70-1.10)$ & 0.3 & $0.98(0.84-1.14)$ & 0.8 \\
\hline $\begin{array}{l}\text { Short term } \\
\text { (less than a year) }\end{array}$ & $55 / 223$ & $1.10(0.81-1.48)$ & 0.6 & $34 / 209$ & $0.69(0.47-1.00)$ & 0.05 & $0.91(0.72-1.15)$ & 0.4 \\
\hline Long term (at least a year) & $86 / 344$ & $1.04(0.81-1.33)$ & 0.8 & $70 / 287$ & $1.02(0.77-1.34)$ & 0.9 & $1.03(0.86-1.24)$ & 0.8 \\
\hline Prostate, risedronate & Trend & & 0.9 & Trend & & 0.3 & Trend & 0.4 \\
\hline Any use & $99 / 455$ & $0.98(0.77-1.23)$ & 0.8 & $70 / 374$ & $0.87(0.66-1.13)$ & 0.3 & $0.93(0.78-1.10)$ & 0.4 \\
\hline $\begin{array}{l}\text { Short term } \\
\text { (less than a year) }\end{array}$ & $38 / 208$ & $0.83(0.58-1.18)$ & 0.3 & $32 / 165$ & $0.90(0.61-1.33)$ & 0.6 & $0.86(0.66-1.12)$ & 0.3 \\
\hline Long term (at least a year) & $61 / 247$ & $1.09(0.81-1.46)$ & 0.6 & $38 / 209$ & $0.83(0.58-1.19)$ & 0.3 & $0.98(0.78-1.23)$ & 0.8 \\
\hline Lung, alendronate & Trend & & 0.5 & Trend & & 0.4 & Trend & 0.3 \\
\hline Any use & $696 / 2542$ & $1.00(0.89-1.12)$ & 0.9 & $756 / 2701$ & $1.05(0.94-1.18)$ & 0.4 & $1.03(0.95-1.11)$ & 0.5 \\
\hline $\begin{array}{l}\text { Short term } \\
\text { (less than a year) }\end{array}$ & $339 / 1114$ & $1.07(0.92-1.24)$ & 0.4 & $386 / 1220$ & $1.16(1.01-1.34)$ & 0.04 & $1.12(1.01-1.24)$ & 0.04 \\
\hline Long term (at least a year) & $357 / 1428$ & $0.93(0.80-1.08)$ & 0.3 & $370 / 1481$ & $0.96(0.83-1.11)$ & 0.6 & $0.95(0.85-1.05)$ & 0.3 \\
\hline Lung, etidronate & Trend & & 0.8 & Trend & & 0.1 & Trend & 0.2 \\
\hline Any use & $338 / 1262$ & $1.04(0.90-1.20)$ & 0.6 & $356 / 1200$ & $1.13(0.98-1.31)$ & 0.09 & $1.09(0.98-1.20)$ & 0.2 \\
\hline $\begin{array}{l}\text { Short term } \\
\text { (less than a year) }\end{array}$ & $139 / 500$ & $1.02(0.82-1.27)$ & 0.9 & $161 / 502$ & $1.15(0.94-1.42)$ & 0.2 & 1.09 (0.94-1.27) & 0.3 \\
\hline Long term (at least a year) & $199 / 762$ & $1.05(0.87-1.26)$ & 0.6 & $195 / 698$ & $1.12(0.93-1.35)$ & 0.2 & $1.08(0.95-1.24)$ & 0.2 \\
\hline Lung, risedronate & & & 0.9 & Trend & & 0.7 & Trend & 0.7 \\
\hline Any use & $261 / 848$ & $1.02(0.86-1.21)$ & 0.8 & $252 / 829$ & $1.11(0.94-1.31)$ & 0.2 & $1.06(0.94-1.20)$ & 0.3 \\
\hline $\begin{array}{l}\text { Short term } \\
\text { (less than a year) }\end{array}$ & $125 / 380$ & $1.05(0.83-1.33)$ & 0.7 & $126 / 412$ & $1.06(0.85-1.34)$ & 0.6 & $1.06(0.90-1.25)$ & 0.5 \\
\hline Long term (at least a year) & $136 / 468$ & $0.98(0.78-1.22)$ & 0.8 & $126 / 417$ & $1.13(0.90-1.42)$ & 0.3 & 1.05 (0.89-1.23) & 0.6 \\
\hline
\end{tabular}


pancreatic cancer cells have been shown in vitro (Tassone et al, 2003) and in vivo (Takiguchi et al, 2012). No duration relationship suggests, however, that as with breast and prostate cancers bisphosphonates might inhibit growth only of already existing tumours.

Other cancers. Our study has not shown any significant associations between overall use of bisphosphonates and risk of endometrial, ovarian or cervical cancers, but the numbers for endometrial and cervical cancers were very low and the findings were not consistent across the databases. An association found between ovarian cancer and risedronate use was found only in QResearch data and without a duration relationship. Previous much smaller studies have suggested a possible decreased risk for bisphosphonate users for ovarian (AHR: 0.64, 95\% CI: 0.40-1.03) and endometrial cancers (AHR: 0.64, 95\% CI: 0.38-1.08 (Cardwell et al, 2012) and AOR: 0.7, 95\% CI: 0.4-1.2 (Fortuny et al, 2009)), but the results also did not reach a statistically significant level. Our finding of a decreased risk for bladder cancer, which was not however statistically significant, was consistent with a previous study (AHR: 0.67, 95\% CI: 0.41-1.10, P=0.11) (Cardwell et al, 2012). No statistically significant associations between bisphosphonate use and melanoma risk were found, similar to the earlier study (AHR: 0.80, 95\% CI: 0.53-1.20) (Cardwell et al, 2012).

\section{CONCLUSION}

We have conducted a series of large population-based case-control studies in two primary-care databases examining the association of bisphosphonates with risks of common cancers in the general population and found associations with reduced risks for breast, prostate and pancreatic cancers, but with no duration relationship and only in short-term users. A duration-dependent reduced risk associated with alendronate use was found for prostate cancer, but only in the QResearch data. This study does not provide enough evidence to conclude that bisphosphonates have protective effects on cancer, but the results are reassuring regarding the safety of bisphosphonates.

\section{ACKNOWLEDGEMENTS}

We acknowledge the contribution of EMIS and the University of Nottingham for expertise in creating and maintaining QResearch and to the EMIS practices, which contribute data without whom this research would not be possible.

\section{REFERENCES}

Bangalore S, Kumar S, Kjeldsen SE, Makani H, Grossman E, Wetterslev J, Gupta AK, Sever PS, Gluud C, Messerli FH (2011) Antihypertensive drugs and risk of cancer: network meta-analyses and trial sequential analyses of 324,168 participants from randomised trials. Lancet Oncol 12(1): 65-82.

British Medical Association and Royal Pharmaceutical Society of Great Britain (2008) British National Formulary Vol. 56. British Medical Association and Royal Pharmaceutical Society of Great Britain: London, UK.

Burbos N, Musonda P, Giarenis I, Shiner AM, Giamougiannis P, Morris EP, Nieto JJ (2010) Predicting the risk of endometrial cancer in postmenopausal women presenting with vaginal bleeding: the Norwich DEFAB risk assessment tool. Br J Cancer 102(8): 1201-1206.

Cardwell CR, Abnet CC, Veal P, Hughes CM, Cantwell MM, Murray LJ (2012) Exposure to oral bisphosphonates and risk of cancer. Int J Cancer 131(5): E717-E725.

Chang ET, Smedby KE, Hjalgrim H, Glimelius B, Adami H-O (2006) Reliability of self-reported family history of cancer in a large case-control study of lymphoma. J Natl Cancer Inst 98(1): 61-68.
Chen Z, Arendell L, Aickin M, Cauley J, Lewis CE, Chlebowski R (2008) Hip bone density predicts breast cancer risk independently of Gail score. Cancer 113(5): 907-915.

Chlebowski RT, Chen Z, Cauley JA, Anderson G, Rodabough RJ, McTiernan A, Lane DS, Manson JE, Snetselaar L, Yasmeen S, O'Sullivan MJ, Safford M, Hendrix SL, Wallace RB (2010) Oral bisphosphonate use and breast cancer incidence in postmenopausal women. J Clin Oncol 28(22): 3582-3590.

Corley DA, Kubo A, Zhao W, Quesenberry C (2010) Proton pump inhibitors and histamine-2 receptor antagonists are associated with hip fractures among at-risk patients. Gastroenterology 139(1): 93-101.

Coussens LM, Werb Z (2002) Inflammation and cancer. Nature 420(6917): $860-867$.

Croucher P, Jagdev S, Coleman R (2003) The anti-tumor potential of zoledronic acid. Breast 12(Suppl 2): S30-S36.

Ferlay J, Shin H-R, Bray F, Forman D, Mathers C, Parkin DM (2010) Estimates of worldwide burden of cancer in 2008: GLOBOCAN 2008. Int J Cancer 127(12): 2893-2917.

Fortuny J, Sima C, Bayuga S, Wilcox H, Pulick K, Faulkner S, Zauber AG, Olson SH (2009) Risk of endometrial cancer in relation to medical conditions and medication use. Cancer Epidemiol Biomarkers Prev 18(5): 1448-1456.

Fournier P, Boissier S, Filleur S, Guglielmi J, Cabon F, Colombel M, Clézardin P (2002) Bisphosphonates inhibit angiogenesis in vitro and testosteronestimulated vascular regrowth in the ventral prostate in castrated rats. Cancer Res 62(22): 6538-6544.

Gnant M (2010) Can oral bisphosphonates really reduce the risk of breast cancer in healthy women? J Clin Oncol 28(22): 3548-3551.

Gonzalez-Perez A, Garcia Rodriguez L, Lopez-Ridaura R (2003) Effects of non-steroidal anti-inflammatory drugs on cancer sites other than the colon and rectum: a meta-analysis. BMC Cancer 3(1): 28.

Green J, Czanner G, Reeves G, Watson J, Wise L, Beral V (2010) Oral bisphosphonates and risk of cancer of oesophagus, stomach, and colorectum: case-control analysis within a UK primary care cohort. BMJ 341: c4444.

Guise TA (2008) Antitumor effects of bisphosphonates: promising preclinical evidence. Cancer Treat Rev 34(Suppl 1): S19-S24.

Hecht SS (2008) Etiology of cancer: tobacco. In DeVita, Hellman, and Rosenberg's Cancer: Principles \& Practice of Oncology, Vol. 1, Chapter 11 pp 147-155. Wolters Kluwer/Lippincott Williams \& Wilkins: Philadelphia, PA, USA.

Henderson KD, Bernstein L (2008) Etiology of cancer: obesity and physical activity. In DeVita, Hellman, and Rosenberg's Cancer: Principles \& Practice of Oncology, Vol. 1, Chapter 18pp 239-244. Wolters Kluwer/Lippincott Williams \& Wilkins: Philadelphia, PA, USA.

Herrett E, Thomas SL, Schoonen WM, Smeeth L, Hall AJ (2010) Validation and validity of diagnoses in the General Practice Research Database: a systematic review. Br J Clin Pharmacol 69(1): 4-14.

Hippisley-Cox J, Coupland C (2009) Predicting risk of osteoporotic fracture in men and women in England and Wales: prospective derivation and validation of QFractureScores. BMJ 339: b4229.

Hippisley-Cox J, Coupland C (2010) Unintended effects of statins in men and women in England and Wales: population based cohort study using the QResearch database. BMJ 340: c2197.

Hippisley-Cox J, Hammersley V, Pringle M, Coupland C, Crown N, Wright L (2004) How useful are General Practice databases for research? Analysis of their accuracy and completeness in one research network. Health Inform $J$ 10: $91-109$.

Jick SS, Kaye JA, Vasilakis-Scaramozza C, Rodríguez LAG, Ruigómez A, Meier CR, Schlienger RG, Black C, Jick H (2003) Validity of the General Practice Research Database. J Hum Pharmacol Drug Ther 23(5): 686-689.

Kanis J, McCloskey E, Johansson H, Strom O, Borgstrom F, Oden A (2008) Case finding for the management of osteoporosis with FRAX ${ }^{\circledR}$ assessment and intervention thresholds for the UK. Osteoporos Int 19: 1395-1408.

Mai PL, Garceau AO, Graubard BI, Dunn M, McNeel TS, Gonsalves L, Gail MH, Greene MH, Willis GB, Wideroff L (2011) Confirmation of family cancer history reported in a population-based survey. J Natl Cancer Inst 103(10): 788-797.

Mauck KF, Clarke BL (2006) Diagnosis, screening, prevention, and treatment of osteoporosis. Mayo Clin Proc 81(5): 662-672.

McGlynn KA, Gridley G, Mellemkjaer L, Brinton LA, Anderson KC, Caporaso NE, Landgren O, Olsen JH (2008) Risks of cancer among a cohort of 23,935 men and women with osteoporosis. Int J Cancer 122(8): 1879-1884. 
Mocellin S (2011) Vitamin D and cancer: deciphering the truth. Biochim Biophys Acta 1816(2): 172-178.

National Institute for Health and Clinical Excellence (2008a) Alendronate, etidronate, risedronate, strontium ranelate and raloxifen for preventing bone fractures in postmenopausal women with osteoporosis who have not had a fracture. Information About NICE Technology Appraisal Guidance. p 160.

National Institute for Health and Clinical Excellence (2008b) Alendronate, etidronate, risedronate, strontium ranelate and teriparatide for preventing bone fractures in postmenopausal women with osteoporosis who have already had a fracture. Information About NICE Technology Appraisal Guidance. p 161.

Newcomb PA, Trentham-Dietz A, Hampton JM (2010) Bisphosphonates for osteoporosis treatment are associated with reduced breast cancer risk. Br J Cancer 102(5): 799-802.

Parker C, Coupland C, Hippisley-Cox J (2010) Antipsychotic drugs and risk of venous thromboembolism: nested case-control study. BMJ 341: c4245.

Rennert G, Pinchev M, Rennert HS (2010) Use of bisphosphonates and risk of postmenopausal breast cancer. J Clin Oncol 28(22): 3577-3581.

Royston P (2004) Multiple imputation of missing values. Stata J 4(3): $227-241$.

Royston P (2005) Multiple imputation of missing values: update of ice. Stata J 5(4): 527-536.

Schütze M, Boeing H, Pischon T, Rehm J, Kehoe T, Gmel G, Olsen A, Tjønneland AM, Dahm CC, Overvad K, Clavel-Chapelon F, BoutronRuault M-C, Trichopoulou A, Benetou V, Zylis D, Kaaks R, Rohrmann S, Palli D, Berrino F, Tumino R, Vineis P, Rodríguez L, Agudo A, Sánchez M-J, Dorronsoro M, Chirlaque M-D, Barricarte A, Peeters PH, van Gils $\mathrm{CH}$, Khaw K-T, Wareham N, Allen NE, Key TJ, Boffetta P, Slimani N, Jenab M, Romaguera D, Wark PA, Riboli E, Bergmann MM (2011) Alcohol attributable burden of incidence of cancer in eight European countries based on results from prospective cohort study. BMJ 342: d1584.
Takiguchi S, Nishino Y, Inoue K, Ikeda M, Kataoka Y, Matsusue K, Nishiyama K, Iguchi H (2012) The bisphosphonate incadronate inhibits intraperitoneal dissemination in an in vivo pancreatic cancer model. Oncol Rep 28(1): 111-116.

Tassone P, Tagliaferri P, Viscomi C, Palmieri C, Caraglia M, D'Alessandro A, Galea A, Goel A, Abbruzzese A, Boland CR, Venuta S (2003) Zoledronic acid induces antiproliferative and apoptotic effects in human pancreatic cancer cells in vitro. Br J Cancer 88(12): 1971-1978.

Thomas E, Brewster DH, Black RJ, Macfarlane GJ (2000) Risk of malignancy among patients with rheumatic conditions. Int J Cancer 88(3): 497-502.

Tuomela J, Valta M, Vaananen K, Harkonen P (2008) Alendronate decreases orthotopic PC-3 prostate tumor growth and metastasis to prostatedraining lymph nodes in nude mice. BMC Cancer 8(1): 81 .

Vestergaard P, Fischer L, Mele M, Mosekilde L, Christiansen P (2011) Use of bisphosphonates and risk of breast cancer. Calcif Tissue Int 88(4): 255-262.

Vincent A, Herman J, Schulick R, Hruban RH, Goggins M (2011) Pancreatic cancer. Lancet 378(9791): 607-620.

Vinogradova Y, Coupland C, Hippisley-Cox J (2012) Exposure to bisphosphonates and risk of cancer: a protocol for nested case-control studies using the QResearch primary care database. BMJ Open 2(1): e000548.

Walker AJ, Card T, Bates TE, Muir K (2011) Tricyclic antidepressants and the incidence of certain cancers: a study using the GPRD. Br J Cancer 104(1): 193-197.

Watts NB, Diab DL (2010) Long-term use of bisphosphonates in osteoporosis. $J$ Clin Endocrinol Metab 95(4): 1555-1565.

(c) (1) (2) This work is licensed under the Creative Commons cc) Attribution-NonCommercial-Share Alike 3.0 Unported License. To view a copy of this license, visit http://creativecommons. org/licenses/by-nc-sa/3.0/ 